\title{
Scaling and self-similarity in an unforced flow of inviscid fluid trapped inside a viscous fluid in a Hele-Shaw cell
}

\author{
Arkady Vilenkin ${ }^{1}$, Baruch Meerson ${ }^{1}$, and Pavel V. Sasorov ${ }^{2}$ \\ ${ }^{1}$ Racah Institute of Physics, Hebrew University of Jerusalem, Jerusalem 91904, Israel and \\ ${ }^{2}$ Institute of Theoretical and Experimental Physics, Moscow 117218, Russia
}

\begin{abstract}
We investigate quasi-two-dimensional relaxation, by surface tension, of a long straight stripe of inviscid fluid trapped inside a viscous fluid in a Hele-Shaw cell. Combining analytical and numerical solutions, we describe the emergence of a self-similar dumbbell shape and find non-trivial dynamic exponents that characterize scaling behavior of the dumbbell dimensions.
\end{abstract}

PACS numbers: 47.15.Gf, 47.15.Hg, 47.20.Ky, 47.11.+j

Introduction. Consider a bubble of low-viscosity fluid (say, water) trapped inside a high-viscosity fluid (say, oil) in a quasi-two-dimensional Hele-Shaw cell. What will happen to the shape of the bubble, if the (horizontal) plates are perfectly smooth, and the fluids are immiscible? The answer depends on the initial bubble shape. A perfectly circular bubble (or an infinite straight stripe) will not change, while a bubble of any other shape will undergo surface-tension-driven relaxation until it either becomes a perfect circle, or breaks into two or more bubbles, which then become perfect circles. The bubble shape relaxation is non-local, as it is mediated by a viscous flow in the outer fluid. The resulting free boundary problem is hard for analysis. This is especially true when the bubble has a complex (even fractal) shape, like that observed, in radial geometry, in a strongly forced HeleShaw flow, when the viscous fluid was initially displaced by the inviscid fluid [1]. The shape complexity results from the viscous fingering instability [2, 3]. The forced Hele-Shaw flow is a celebrated problem in fluid dynamics and nonlinear dynamics [4, 5, 6, 7]. The role of small surface tension there is to introduce a (nontrivial) regularization on small scales. This Letter deals with an unforced Hele-Shaw (UHS) problem, where surface tension is the only driving mechanism. We address the UHS problem in the case when the inviscid fluid is initially in the form of a long stripe. We show that this special initial condition provides a useful characterization of the UHS model, as the evolving stripe, which develops a dumbbell shape, exhibits self-similarity with non-trivial dynamic exponents.

UHS problem. Let the inner fluid have negligible viscosity, so that the pressure inside the bubble is homogeneous. The velocity of the viscous outer fluid is $\mathbf{v}(\mathbf{r}, t)=-\left(b^{2} / 12 \mu\right) \nabla p(\mathbf{r}, t)$, where $p$ is the pressure, $\mu$ is the dynamic viscosity, and $b$ is the plate spacing $[2,[3,4,5]$. Therefore, the interface speed is

$$
v_{n}=-\left(b^{2} / 12 \mu\right) \nabla_{n} p
$$

where index $n$ denotes the components of the vectors normal to the interface, and $\nabla_{n} p$ is evaluated at the respective points of the interface $\gamma$. In view of incompressibility of the outer fluid, the pressure is a harmonic function:

$$
\nabla^{2} p=0 .
$$

The Gibbs-Thomson relation at the interface yields

$$
\left.p\right|_{\gamma}=(\pi / 4) \sigma \mathcal{K},
$$

where $\sigma$ is surface tension, and $\mathcal{K}$ is the local curvature of the interface, positive when the inviscid region is convex outwards. As both the supply of the inner fluid, and evacuation of the outer fluid are blocked, we demand

$$
\left.\nabla_{n} p\right|_{\Gamma}=0
$$

at the external boundary of the system $\Gamma$. Equations (11)-(41) define the exterior UHS problem (see Ref. 8] for a more detailed discussion). A related, but different interior problem has been also considered, mainly in the context of singularity formation (pinch-offs) in bubbles of viscous fluid [9]. The UHS model has two important properties: (i) the bubble area remains constant, (ii) the length of the interface between the two fluids is a nonincreasing function of time 10 .

The UHS problem is not integrable. Moreover, we are unaware of any analytical solutions to this problem, except for a linear analysis of a slightly deformed flat or circular interface 11]. Owing to its two-dimensionality, the problem can be reformulated as a nonlocal nonlinear partial differential equation for a conformal map which is analytic in the exterior of the unit circle 12]. This equation, however, is hard for analysis. We consider here a simple but non-trivial case that can be analyzed directly in the physical plane: the dynamics of a half-infinite (or, physically, very long) stripe.

Stripe dynamics: theoretical predictions. Let at $t=$ 0 the bubble have the form of a half-infinite straight stripe of width $\Delta$, located along the $x$-axis as shown in Fig. [1 The external boundary of the system $\Gamma$ is at infinity, where the pressure is bounded. We will measure the distance in units of $\Delta$, the time in units of $\tau=48 \mu \Delta^{3} /\left(\pi \sigma b^{2}\right)$, and the pressure in units of $p_{0}=\pi \sigma /(4 \Delta)$. In the rescaled variables Eqs. (1) and (3) become $v_{n}=-\nabla_{n} p$ and $\left.p\right|_{\gamma}=\mathcal{K}$, so the rescaled problem is parameter-free. 


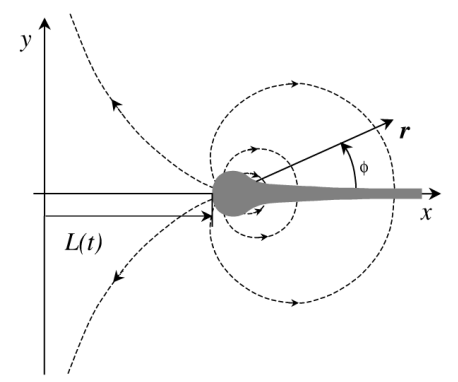

FIG. 1: Setting for the dumbbell dynamics.

We are interested in the late-time behavior: $t \gg 1$. Because of the Gibbs-Thomson effect, the pressure gradient is largest near the tip, so the tip retreats along the $\mathrm{x}$-axis. As the bubble area must be conserved, the retreating stripe acquires a dumbbell shape, and the lobe of the dumbbell expands with time, see Fig. 2] Surprisingly, the main contribution to the dumbbell area comes, at $t \gg 1$, from the dumbbell neck, and not from the lobe.

Going over to a quantitative analysis, we assume (and later verify numerically) that the lobe can be characterized by a single time-dependent length scale $R(t)$. Another length scale is $L(t)$ : the retreat distance of the dumbbell. Our main objective is to find the exponents of the power laws for $R(t)$ and $L(t)$. Our analysis will not give the numerical coefficients of these power laws (which, in the rescaled units, are of order unity); these will be found numerically. Introduce polar coordinates $r$ and $\phi$, see Fig. 1 The dumbbell neck, $r \gg R(t)$, is almost flat, so $p$ must vanish at $\phi \rightarrow 0$ and $\phi \rightarrow 2 \pi$. On the other hand, $p=\mathcal{K} \sim 1 / R(t)$ at the lobe interface (for definiteness, at $\phi= \pm \pi / 2$ ). Therefore, the leading term in the multipole expansion [13] is

$$
p(r, \phi, t)=C[R(t) r]^{-1 / 2} \sin (\phi / 2),
$$

where $C=\mathcal{O}(1)$. Having demanded the Gibbs-Thomson condition here, we somewhat stretched the validity of Eq. (5D), but this can only affect the value of constant $C$. The dashed lines in Fig. 1 1 show the field lines of $\nabla p$.

Equation (5) yields the normal component of the interface speed $v_{n}=-\nabla_{n} p$ in the neck region. For the upper interface of the neck

$$
v_{n}=-\frac{1}{r} \frac{\partial p}{\partial \phi}(\phi \rightarrow 0)=-\frac{C}{2 R^{1 / 2}(t) r^{3 / 2}} .
$$

Now we return to the Cartesian coordinates. Let $h\left(x_{1}, t\right)$ be the local height of the dumbbell, while $x_{1}=x-L(t)$ be the horizontal coordinate in the moving frame with the origin at the tip. In the neck region, $x_{1} \gg R(t)$, the quantity $\partial h\left(x_{1}, t\right) / \partial t$ is given by Eq. (6), so we obtain

$$
h\left(x_{1}, t\right)-\frac{1}{2}=\int_{0}^{t} \frac{C d t^{\prime}}{2 R^{1 / 2}\left(t^{\prime}\right) x_{1}^{3 / 2}} .
$$

This equation yields $h\left(x_{1}, t\right)$ in two different limits. At very large distances from the lobe, $x \gg L(t)$ (region I)

$$
h\left(x_{1}, t\right)-\frac{1}{2} \simeq \frac{C}{2 x_{1}^{3 / 2}} \int_{0}^{t} \frac{d t^{\prime}}{R^{1 / 2}\left(t^{\prime}\right)} \sim \frac{t}{x_{1}^{3 / 2} R^{1 / 2}(t)},
$$

where the last estimate assumes that $R(t)$ is a power of $t$. Another limit corresponds to intermediate distances: $R(t) \ll x_{1} \ll L(t)$ (region II). Here, at fixed $x$, the main contribution to the integral in Eq. (7) comes from times close to $t$, so that $x_{1}(t) / \dot{L}(t) \ll t-t^{\prime} \ll t$. Indeed, one can expand $x_{1}\left(t^{\prime}\right)=x_{1}(t)+\dot{L}(t)\left(t-t^{\prime}\right)+\ldots$ and, in the leading order, ignore higher order terms. The effective time interval for the integration is $\left(t-\delta t^{\prime}, t\right)$, where $\delta t^{\prime} \sim x_{1}(t) / \dot{L}(t)$. Furthermore, $R^{1 / 2}\left(t^{\prime}\right)$ can be evaluated at $t^{\prime}=t$, as its variation on the time interval $\left(t-\delta t^{\prime}, t\right)$ is negligible. Then, extending the lower limit of the integral to $-\infty$ and calculating the remaining elementary integral, we obtain

$$
h\left(x_{1}, t\right)-\frac{1}{2} \simeq \frac{C}{R^{1 / 2}(t) \dot{L}(t) x_{1}^{1 / 2}(t)} .
$$

Now we can estimate the contributions of regions I and II to the dumbbell area gain $A$ in the neck region. We integrate Eq. (8) over $x_{1}$ from, say, $2 L(t)$ to infinity, and Eq. (9) from $R(t)$ to $2 L(t)$. The results are:

$$
A_{I}(t) \sim \frac{t}{L^{1 / 2}(t) R^{1 / 2}(t)} \quad \text { in region I, }
$$

and

$$
A_{I I}(t) \sim \frac{L^{1 / 2}(t)}{\dot{L}(t) R^{1 / 2}(t)} \quad \text { in region II } .
$$

Once $L(t)$ is a power law, $A_{I}$ and $A_{I I}$ are comparable. Notice that in region I (respectively, II) the main contribution comes from the lower (respectively, upper) limit of integration. As we verify a posteriori, the contribution to the dumbbell area of the lobe itself, $A_{R} \sim R^{2}(t)$, is negligible compared to $A_{I}$ and $A_{I I}$ as long as $t \gg 1$.

Now we can find the dynamic exponents of $L(t)$ and $R(t)$. First, we employ the area conservation of the dumbbell. The area loss $L(t) \times 1$ of the retreating dumbbell must be equal to the area gain in the neck, so up to numerical coefficients of order unity

$$
L(t) \sim A_{I}(t) \sim A_{I I}(t) \sim \frac{t}{L^{1 / 2}(t) R^{1 / 2}(t)} .
$$

Second, there is a simple kinematic relation between $\dot{L}(t)$ and the characteristic speed of the lobe motion $V_{l}$. Using Eq. (5), we obtain $V_{l} \sim-\partial p / \partial r(r \sim R(t), \phi \simeq \pi) \sim$ $R^{-2}(t)$, and demand $\dot{L}(t) \sim R^{-2}(t)$. Combined with Eq. (12), this yields

$$
L(t) \sim t^{3 / 5} \text { and } R(t) \sim t^{1 / 5} \text { at } t \gg 1 .
$$


Now we can return to Eqs. (5)-(11) and find the explicit time-dependences. For example, the far-neck asymptote in Eq. (8) becomes $h(x, t)-1 / 2 \sim t^{9 / 10} x^{-3 / 2}$. We can also verify that, at $t \gg 1$, the lobe area $A_{R} \sim R^{2}(t) \sim$ $t^{2 / 5}$ is indeed much less than $A_{I}(t) \sim A_{I I}(t) \sim t^{3 / 5}$.

That the lobe is characterized by a single dynamic length scale $R(t) \sim t^{1 / 5}$ implies a similarity Ansatz for the lobe shape in the moving frame:

$$
h_{s}\left(x_{1}, t\right)=t^{1 / 5} \Phi\left(x_{1} / t^{1 / 5}\right) \quad \text { at } t \gg 1 .
$$

Numerical method. To test our predictions, we performed simulations of the dynamics of long stripes with dimensions $X \times 1$, where $X \gg 1$. The ultimate shape of such a stripe is a perfect circle. Therefore, the scaling behavior, predicted by our theory of a one-sided dumbbell, appears as an intermediate asymptote, as we require $R(t) \gg 1$ but $L(t) \ll X$. In view of the predicted scalings with time, we must demand $1 \ll t \ll X^{5 / 3}$.

Our numerical algorithm [14] is based on a representation of the harmonic field in terms of a line integral over the bounding contour; it involves tracking of the contour nodes. We employed a variant of the boundary integral method, suggested in Ref. 15]. The algorithm includes solving an integral equation for an effective density of dipole moments (DMD) and evaluating another integral, which yields a harmonic conjugated function $(\mathrm{HCF})$. The normal velocity of the interface is given by the derivative of the HCF along the contour. The very large aspect ratio of the dumbbell demands a different discretization compared to Ref. 15]. Indeed, the typical scale of variation of the kernel of the integral equation 15. over the almost flat neck of the dumbbell is close to 1: the initial stripe thickness. On the other hand, the DMD changes much slower there. This enabled us to considerably reduce the number of grid nodes in the neck region. We used a piecewise constant function to approximate the $\mathrm{DMD}$, and a piecewise linear function to approximate the contour. Therefore, each of the integrals was approximated as a discrete sum of the DMD values multiplied by an integral of the kernel between two neighboring nodes. The latter integrals can be calculated analytically. The $\mathrm{HCF}$ is evaluated at middle points between the nodes, while the normal velocity at each node is evaluated using the values of the HCF at neighboring middle points.

We used an explicit finite difference method to track the contour. The number of grid points, needed for an accurate solution and contour tracking, decreases with time together with the perimeter of the dumbbell. An obvious modification of the algorithm of Ref. [15] exploited the 4-fold symmetry of the dumbbell. The area conservation of the dumbbell was used for accuracy control. The time step chosen was $5 \times 10^{-3} \min \left|R^{i} / v_{n}^{i}\right|$, where $R_{i}$ and $v_{n}^{i}$ are the local curvature radius and normal velocity, respectively, in the node $i$ of the contour. This choice resulted in good area conservation: in the simulation described below less than $0.5 \%$ of the area

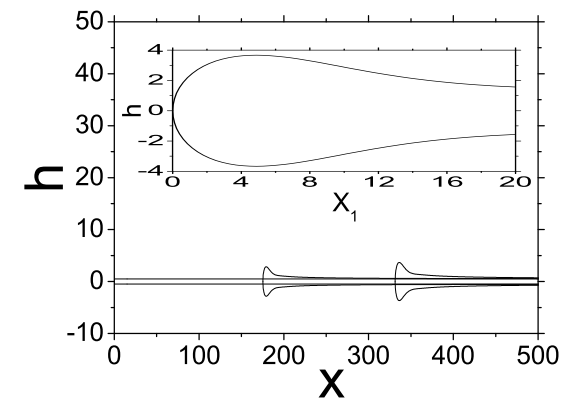

FIG. 2: Snapshots of a part of the simulated system at $t=$ 0,1000 and 3010. Notice the large difference between the horizontal and vertical scales. The inset shows, to scale, the lobe region at $t=3010$.
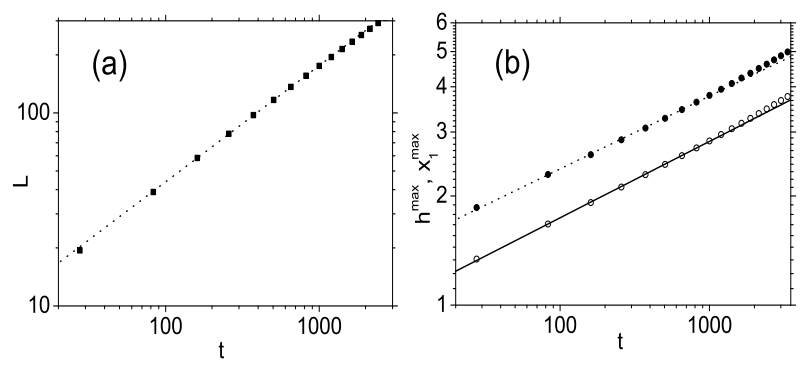

FIG. 3: Figure a shows, in a $\log -\log$ scale, the retreat distance $L$ versus time and its power-law fit $2.75 t^{0.60}$. Figure b shows, in a log-log scale, the maximum dumbbell height, $h^{\max }$ (the empty circles), and the position of the maximum, $x_{1}^{\max }$ (the filled circles), versus time, as well as their power-law fits $0.66 t^{0.21}$ and $0.94 t^{0.20}$, respectively.

was lost by the time $t=7000$. As the dumbbell contour becomes smoother, the time step greatly increases.

Numerical results. Here we report a simulation with $X=2000$. Figure 2 shows snapshots of a part of the system at times $t=0,1000$ and 3010. The stripe develops a dumbbell shape (though some may prefer a comparison with daisy petal). The lobe grows with time, the neck widens. Shown in Fig. $3 \mathrm{k}$ is the retreat distance $L(t)$ versus time. A power law fit yields exponent 0.60 which coincides with the theoretical value $3 / 5$. Figure $3 \mathrm{~b}$ shows the maximum dumbbell height, $h^{\max }$, and the position of the maximum, $x_{1}^{\max }$, versus time. Each of these two quantities exhibits a power law; the fitted exponents are 0.21 (for $h^{\max }$ ) and 0.20 (for $x_{1}^{\max }$ ), in agreement with the theoretical value $1 / 5$. At long times, when the aspect ratio of the dumbbell is already not large enough, the straight line in Fig. 3 a slightly curves down, while those in Fig. 3b curve up. We verified that for a shorter stripe, $X=1000$, deviations from the same straight lines occur earlier, as expected. The time interval of the three fits, $20<t<1000$, corresponds to the common parts of the dependences for the two values of $X$.

Figure 4 depicts the (rescaled) dumbbell shape in the moving frame at three different times. The collapse of 


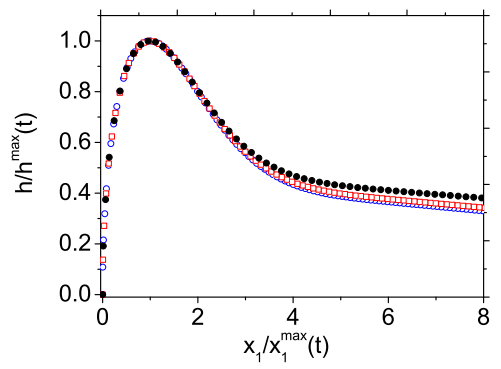

FIG. 4: Self-similarity of the lobe. Shown is the shape function $h\left(x_{1}, t\right)$, rescaled to the maximum dumbbell elevation, versus the coordinate $x_{1}$, rescaled to the abscissa of the maximum, at times 160.3 (the filled circles), 1000 (the squares), and 3010 (the empty circles).
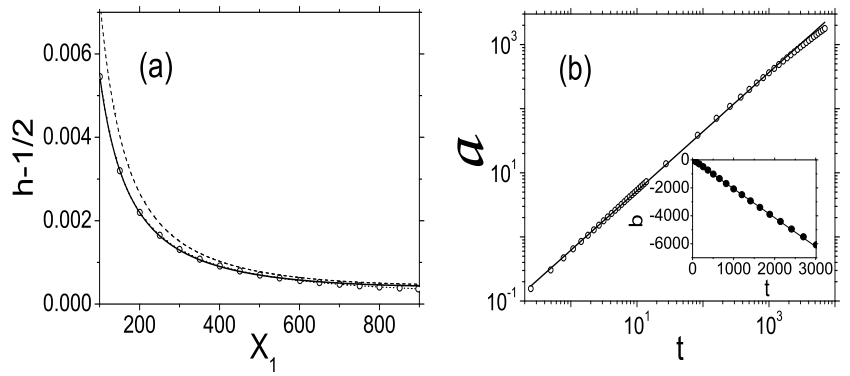

FIG. 5: The dumbbell neck shape and dynamics. Figure a: the neck shape at $t=13.9$, found numerically (the dotted line with circles), Eq. (15) (the solid line), and the first term of Eq. (15) (the dashed line). Figure $b$ and its inset show, in a $\log$-log and linear scales, respectively, the pre-factors $a$ and $b$ versus time (symbols). Also shown are a power-law fit with exponent 0.92 (figure b) and a linear fit (inset).

three different curves into a single one supports the similarity Ansatz (14). Notice that the dumbbell shape $h\left(x_{1}, t\right)$ in region II $\left[R(t) \ll x_{1} \ll L(t)\right]$ belongs to the similarity region. Indeed, assuming that $\Phi(\xi) \sim \xi^{-1 / 2}$ at $\xi \gg 1$, we see that Eq. (14) yields Eq. (9) (where one should substitute $L \sim t^{3 / 5}$ and $R \sim t^{1 / 5}$, and neglect $1 / 2$ in the left hand side).

The self-similarity breaks down at a distance $x_{1} \sim$ $L(t) \sim t^{3 / 5}$ from the tip. Beyond this distance, Eq. (8) predicts a power-law neck shape. Figure 5 shows the shape of the dumbbell neck at time $t=13.9$, computed numerically. Also shown are the quantity

$$
\begin{array}{r}
h\left(x_{1}\right)-\frac{1}{2}=a\left[x_{1}^{-3 / 2}+\left(X-2 L-x_{1}\right)^{-3 / 2}\right]+ \\
b\left[x_{1}^{-2}+\left(X-2 L-x_{1}\right)^{-2}\right],
\end{array}
$$

and its first term, proportional to $a$. Equation (15) differs from Eq. (8) in that (i) it accounts for contributions from two dumbbell lobes, and (ii) it accounts for the subleading term in the multipole expansion of the harmonic function $p$. Note that $a$ and $b$ are the only adjustable parameters in Eq. (15). The resulting profile is almost indistinguishable from the numerical profile. The first term of Eq. (15) already gives fairly good agreement. The excellent agreement holds, on a shrinking interval of $x_{1}$, until $t=7020$. Figure $5 \mathrm{~b}$ and its inset show $a$ and $b$ versus time, respectively. A power-law fit of $a(t)$ yields exponent 0.92 , close to our prediction $9 / 10$. The pre-factor $b(t)$ behaves linearly with time. How does it compare with the theory? As $p \sim 1 / R(t)$ at the lobe interface, the sub-leading term $p \sim r^{-1} \sin \phi$ does not include $R(t)$. Then, repeating the procedure which led us to Eq. (8), we do obtain $b(t) \sim t$.

Summary. We studied the UHS flow in the case when the inviscid fluid is initially in the form of a long stripe. We found that the resulting dumbbell dynamics exhibit self-similarity with nontrivial exponents. The solution we obtained is the first analytical solution for an UHS flow that goes beyond a linear theory. Similarly to other curve-shortening area-preserving relaxation models [16], the stripe relaxation provides a useful characterization of this non-integrable flow. Its experimental realization should not be difficult.

We thank Eran Sharon for a useful discussion. This work was supported by the Israel Science Foundation (Grant No. 180/02), and by the Russian Foundation for Basic Research (Grant No. 05-01-000964).

[1] E. Sharon, M.G. Moore, W.D. McCormick, and H.L. Swinney, Phys. Rev. Lett. 91, 205504 (2003).

[2] P.G. Saffman and G.I. Taylor, Proc. R. Soc. London, Ser. A 245, 312 (1958).

[3] L. Paterson, J. Fluid Mech. 113, 513 (1981).

[4] J.S. Langer, in Chance and Matter, edited by J. Souletie, J. Vannimenus, and R. Stora (Elsevier, Amsterdam, 1987).

[5] D. Bensimon, L.P. Kadanoff, S. Liang, B.I. Shraiman, and C. Tang, Rev. Mod. Phys. 58, 977 (1986).

[6] D.A. Kessler, J. Koplik, and H. Levine, Adv. Physics 37, 255 (1988).

[7] J. Casademunt and F.X. Magdaleno, Phys. Rep. 337, 1 (2000).

[8] M. Conti, A. Lipshtat, and B. Meerson, Phys. Rev. E 69, 031406 (2004).

[9] R. Almgren, Phys. Fluids 8, 344 (1996).

[10] P. Constantin and M. Pugh, Nonlinearity 6, 393 (1993).

[11] The damping rates of small sinusoidal perturbations of flat and circular interfaces are given by the zero-flow-rate limit of Eq. (10) of Ref. [2] (flat interface), and of Eq. (11) of Ref. 3] (circular interface).

[12] P. Constantin and L. Kadanoff, Physica D 47, 450 (1991).

[13] J.D. Jackson, Classical Electrodynamics (Wiley, New York, 1975), p. 76.

[14] A. Vilenkin and B. Meerson, arXiv physics/0512043

[15] A. Greenbaum, L. Greengard, and G.B. McFadden, J. Comput. Phys. 105, 267 (1993).

[16] R. Thouy, N. Olivi-Tran, and R. Jullien, Phys. Rev. B 56, 5321 (1997); A. Peleg, B. Meerson, A. Vilenkin, and M. Conti, Phys. Rev. E 63, 066101 (2001). 